\title{
Low Complexity Algebraic Multicast Network Codes
}

\author{
Sidharth Jaggi ${ }^{1}$ \\ Dept. of Electrical Engineering \\ California institute of Technology \\ Pasadena, CA, USA 91125 \\ e-mail: jaggi@caltech.edu
}

\author{
Philip A. Chou, and Kamal Jain \\ Microsoft Corporation \\ One Microsoft Way \\ Redmond, WA, USA 98052-6399 \\ \{pachou, kamalj\}@microsoft.com
}

\begin{abstract}
We present a low complexity algorithm for designing algebraic codes that achieve the information theoretic capacity for the multicast problem on directed acyclic networks. These codes operate over field sizes which are significantly smaller than those previously known, leading to significantly lower design and implementation complexity, and network link usage. These codes can be extended for networks with cycles and delays, and for robustness properties.

A network is represented by a directed graph $\mathcal{G}=(V, E)$ with a vertex set $V$ and edge set $E$. Multiple edges are allowed between vertices, and $\mathcal{G}$ is acyclic. A source node $s \in V$ generating information at rate $R$, and a set of $N$ receiver nodes $\mathcal{R}=\left\{r_{1}, r_{2}, \ldots, r_{N}\right\} \subset V$, are specified. Nodes are allowed to transmit on outgoing edges any causal function of information on incoming edges. The multicast capacity is defined as the maximum rate at which identical information can be decoded by each node in $\mathcal{R}$. The multicast network coding problem is the 4-tuple $(\mathcal{G}, s, \mathcal{R}, R)$, and it is said to have a solution if there exist causal functions for each node in $V$ such that the multicast capacity equals $R$. The solution comprises these functions at each node in $V$ and the decoders at nodes in $\mathcal{R}$. Let $C \leq \min _{i}\left\{C_{i}\right\}$, where $C_{i}$ is the min-cut max-flow capacity of the network between $s$ and $r_{i}$. For any $\epsilon>0$, the multicast network coding problem $(\mathcal{G}, s, \mathcal{R}, C-\epsilon)$ was shown by a random coding argument in [1] to have a solution. Further,

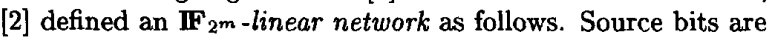
concatenated in blocks of length $m$ to form symbols in the finite field $\mathbf{I F}_{2^{m}}$. Nodes in $V$ perform linear combinations (over $\mathbf{F F}_{2^{m}}$ ) of the symbols on incoming edges to obtain symbols on outgoing edges. They show that for $2^{m}>N C$ there exist

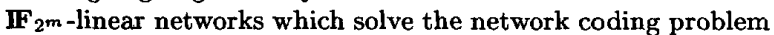
$(\mathcal{G}, s, \mathcal{R}, C)$
\end{abstract}

Our main results, many of which were independently and concurrently discovered by [3], are as follows

Theorem 1 (Network Multicast Algorithm) For $m=$ $\left\lceil\log _{2} N\right\rceil$ and any $R \leq C$, there exist $\mathbf{I F}_{2^{m} \text {-linear networks }}$ which solve the multicast network coding problem $(\mathcal{G}, s, \mathcal{R}, R)$.

Outline of Proof:- We provide a polynomial time construction for the solution. The network is decomposed into $R$ edgedisjoint paths to each $r_{i}$. These paths are then merged using the directed acyclic structure of the network to form the network flow. Linear combinations are designed for this network flow one edge at a time. One ensures that at every step of this design algorithm, for each receiver there exists a set of $R$ edges on edge-disjoint paths to that receiver, such that these edges carry linearly independent combinations of the $R$ symbols generated by $s$ in any coding interval. More details are

${ }^{1}$ The first author was supported by Microsoft Corporation. given in [4]. Decoding at each $r_{i}$ is then the inverse transformation of the linear map taking the symbols generated by the source to the symbols on incoming edges to $r_{i}$.

Theorem 2 (Lower bound on field size) For any $k$ there exist networks $\mathcal{G}$ with multicast capacity 2 with $\left(\begin{array}{c}k+1 \\ 2\end{array}\right)$ receivers, such that for any $2^{m}<k$ there exist no $\mathbf{I F}_{2^{m}}$-linear networks which solve the network coding problem $(\mathcal{G}, s, \mathcal{R}, 2)$.

The proof is constructive (details in [4]). This provides a lower bound to the finite field size required.

Theorem 3 (Robustness) For $m=\left\lceil\log _{2}\left(N\left(\begin{array}{l}C-1 \\ R-1\end{array}\right)\right)\right\rceil$, there

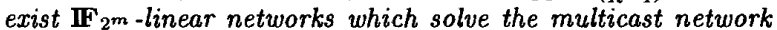
coding problem $\left(\mathcal{G}^{\prime}, s, \mathcal{R}, R\right)$, for any network $\mathcal{G}^{\prime}$ obtained by less than $C-R+1$ edge failures in $\mathcal{G}$.

This generalizes the concept of MDS codes to multicast networks and shows the existence of codes which can tolerate up to $C-R$ network link failures while leaving the linear combinations at the source and internal nodes the same.

We now define the more general concept of $\left(\mathbf{I F}_{2^{m}}\right)^{n}$-linear networks, wherein each edge transmits an $n$-length vector of symbols from $\mathbf{I F}_{2^{m}}$, vectors on incoming edges to a node are concatenated to form a single concatenated input vector, and each node performs a different local linear transformation to its concatenated input vector to obtain an $n$-length vector on each outgoing edge. Using random coding arguments one can prove that randomly chosen $\left(\mathbf{I F}_{2^{m}}\right)^{m}$-linear networks have a high probability of being solvable, and of having strong robustness properties.

Theorem 4 (Randomized Algorithm) Under iid choices of all local linear transformations the probability that the resulting $\left(\mathbf{I F}_{2^{m}}\right)^{n}$-linear network solves the multicast network coding problem $(\mathcal{G}, s, \mathcal{R}, R)>1-2^{\log _{2} N+2-n m(C-R)}$.

Theorem 5 (Randomized Robust Algorithm)

Under iid choices of all local linear transformations the probability that the resulting $\left(\mathbf{F}_{2^{m}}\right)^{n}$-linear network solves the multicast network coding problem $\left(\mathcal{G}^{\prime}, s, \mathcal{R}, R\right)$ $>1-2^{\log _{2} N+2+|E|-n m\left(C^{\prime}-R\right)}$ (where $C^{\prime}$ is the multicast capacity of any $\mathcal{G}^{\prime}$ whose edge-set is a subset of that of $\left.\mathcal{G}\right)$.

REFERENCES

[1] R. Ahlswede, N. Cai, S.-Y. R. Li, and R. W. Yeung, "Network Information Flow", IEEE Transactions on Information Theory, IT-46, pp. 1204-1216, 2000.

[2] R. Koetter, M. Medard, "An Algebraic Approach to Network Coding", presented at INFOCOM 2002.

[3] P. Sanders, S. Egner, L. Tolhuizen, "Polynomial Time Algorithms for Network Information Flow", Proc. of the 15th ACM Symposium on Parallelism in Algorithms and Architectures.

[4] P. A. Chou, M. Effros, S. Egner, S. Jaggi, K. Jain, P. Sanders, L. Tolhuizen, "Linear Multicast Network Coding Algorithms", in preparation for IEEE Transactions on Information Theory. 